\title{
Intracranial Atherosclerosis Burden and Stroke Recurrence for Symptomatic Intracranial Artery Stenosis (sICAS)
}

\author{
Ping Sun 1,7, \#, Liping Liu ${ }^{1,2,3,4, ~ \#, ~ Y u e s o n g ~ P a n ~}{ }^{1,5,6}$, Xianwei Wang ${ }^{1,2,3,4}$, Donghua Mi1,2,3,4, Yuehua \\ $\mathbf{P u}^{1,2,3,4}$, Xin Liu ${ }^{1,2,3,4}$, Wanying Duan ${ }^{1,2,3,4}$, Hongyi Yan ${ }^{1,2,3,4}$, Chunxue Wang ${ }^{1,2,3,4}$, Xingquan \\ Zhao $^{1,2,3,4}$, Yilong Wang ${ }^{1,2,3,4}$, Yongjun Wang ${ }^{1,2,3,4}$, on behalf of CICAS investigators
}

${ }^{1}$ Department of Neurology, Beijing Tiantan Hospital, Capital Medical University, Beijing, China. ${ }^{2}$ China National Clinical Research Center for Neurological Diseases, Beijing, China. ${ }^{3}$ Center of Stroke, Beijing Institute for Brain Disorders, Beijing, China. ${ }^{4}$ Beijing Key Laboratory of Translational Medicine for Cerebrovascular Disease, Beijing, China. ${ }^{5}$ Department of Epidemiology and Health Statistics, School of Public Health, Capital Medical University, Beijing, China. ${ }^{6}$ Beijing Municipal Key Laboratory of Clinical Epidemiology, Beijing, China. ${ }^{7}$ The Second People's Hospital of Guiyang, Guizhou, China. ${ }^{8}$ Department of Medicine and Therapeutics, Prince of Wales Hospital, Chinese University of Hong Kong, Shatin Hong Kong SAR.

[Received February 2, 2018; Revised February 26, 2018; Accepted March 1, 2018]

\begin{abstract}
Intracranial atherosclerosis burden is an arising key index for the risk and prognosis for Intracranial Atherosclerosis Stenosis (ICAS). The present study estimated one-year prognosis for patients of symptomatic ICAS with different degrees of intracranial atherosclerosis burden (ICASB) and identified whether the category of multiple and single acute infarction was associated with atherosclerosis burden. A total of 2864 consecutive patients, from 22 hospitals across China, who experienced an acute cerebral ischemia $<7$ days after onset of symptoms were evaluated. All patients underwent magnetic resonance angiography, and the degree of intracranial stenosis with the ICASB was calculated. The patients were categorized into three groups according to ICASB grading: $<4,4-5$ and $>5$ scores. Multivariate Cox proportional hazards regression models were used to estimate the impact of the hazard ratios(HR) of the putative determinants of recurrent stroke in one year. In the groups with ICASB 4-5 and ICASB >5scores recurrent stroke were significantly higher than the other $(P<0.0001)$. On multivariate logistic analysis, ICASB $(4-5)$ indicated more stroke recurrence at 12 months (adjusted hazard ratio, 1.96; 95\% confidence interval, 1.08-3.56; $P=0.027)$, compared to the ICASB $<4$ scores and $>5$ groups $(P<0.001)$. Moreover, proportion of single and multiple infarction lesions differs with different ICASB. Multiple lesions were related with higher of $\operatorname{ICASB}(\mathbf{P}<0.001)$. Intracranial atherosclerosis burden was associated with recurrent stroke at 12 months. Multiple infarction lesions were associated with higher ICASB score which indicate higher risk of recurrent.
\end{abstract}

Key words: Intracranial Atherosclerosis Burden, ICAS, recurrence

Intracranial atherosclerotic stenosis (ICAS) is a main etiology of ischemic stroke, especially in Asian patients $[1,2]$. Previous studies $[3,4]$ had shown that multiple location of ICAS were independent predictors of unfavorable outcome at 6 months. In addition, the patients exhibiting recent symptoms of intracranial

*Correspondence should be addressed to: Dr. Yongjun Wang, Department of Neurology, Beijing Tiantan Hospital, Capital Medical University, Beijing, China. Email: yongjunwang111@aliyun.com. "Ping Sun and Liping Liu contributed equally to the manuscript.

Copyright: ( 2018 Sun P et al. This is an open-access article distributed under the terms of the Creative Commons Attribution License, which permits unrestricted use, distribution, and reproduction in any medium, provided the original author and source are credited. 
stenosis face a maximal risk of $\geq 70 \%$ of subsequent stroke in the territory of the stenotic artery. The Chinese Intracranial Atherosclerosis (CICAS) study [5] reported that severe stenosis and multiple stenosis are risk factors for recurrent stroke. Moreover, intracranial atherosclerosis burden (ICASB) is regarded as the index more reflective of pathology for ICAS patients. Although the differ outcome of patients with the different degrees of stenosis were observed, but the recruitment in this group were moderate or severe stenosis of $>50 \%$, who could be considered as a group of high recurrent rate.

From the report of the TIAregistry [6], indicated that patients with multiple acute infarction lesions had more than double of stroke recurrence compared to those with single acute infarction. This study aimed to examine the recurrent of ICAS among patients presenting acute ischemic stroke, in order to identify the prognostic value of atherosclerosis burden and related imaging category of lesions.

\section{MATERILAS AND METHODS}

\section{Patients and Workups}

Chinese Intracranial Atherosclerosis (CICAS) is a prospective, multicenter, hospital-based study, encompassing twenty-two general hospitals. The study had been approved by the Institutional Review Board at the participating hospitals, and informed consent was obtained from each participant prior to the study. Clinical and imaging data were prospectively collected from patients with ischemic stroke (IS) or transient ischemic attack. Patients who presented the onset of symptoms in $<7$ days were enrolled in the study and were aged between 18 and 80 years. Patients who were clinically unstable or required close monitoring or were disabled before admission (modified Rankin scale (mRS) score $>2$ ) and were physically or subjectively unable to comply with the magnetic resonance (MR) examination were excluded. Patients with cardio-embolic risk factors (atrial fibrillation, valvar heart disease, postcardiac valve replacement) and undetermined causes were also excluded.

\section{Clinical Information Assessment}

Clinical information included age, sex, and vascular risk factors, such as hypertension (defined by a history of hypertension, or diagnosed at discharge), diabetes mellitus (defined by a history of diabetes mellitus or diagnosis at discharge), hyperlipidemia (defined as lowdensity lipoprotein cholesterol more than or equal to 2.6 $\mathrm{mmol} / \mathrm{L}$ at admission or a history of hyperlipidemia or receiving lipid-lowering treatment or diagnosis at discharge), history of IS or intracerebral hemorrhage, heart disease (defined as a history of myocardial infarction, angina pectoris, and congestive heart failure). National Institutes of Health Stroke Scale (NIHSS) score at admission and discharge, and $\mathrm{mRS}$ score at discharge and 1 year after stroke onset were evaluated. Smoking history, current or previous smokers (continuously smoking $\geq 1$ cigarette a day for 6 months), and a history of heavy alcohol use (drinking $>2 \mathrm{U} /$ day on average for men or $>1 \mathrm{U} /$ day on average for women) was also collected. In addition, we recorded the using of antithrombotic therapy within 48 hours of admission, at discharge and 1 year after stroke onset.

\section{Image Interpretation}

All patients underwent conventional MRI on a 3.0Tor 1.5T MR scanner. The imaging sequences included a 3dimensional time-of-flight MRA (repetition time/echo time (TR/TE), 20-25/3.3-3.9 ms; flip angle, 15-20'; slice thickness, $0.65-1.00 \mathrm{~mm}$ ), T2/T1-weighted imaging (repetition time, $4500 \mathrm{~ms}$; echo time, $84 \mathrm{~ms}$; repetition time, $1200 \mathrm{~ms}$; echo time, $11 \mathrm{~ms}$ ), fluid-attenuated inversion recovery sequences (repetition time, $7000 \mathrm{~ms}$; echo time, $94 \mathrm{~ms}$ ), and diffusion-weighted imaging (repetition time, $3000 \mathrm{~ms}$; echo time, $75 \mathrm{~ms}$ ). Two neurologists interpreted the MRI images. In the event of disagreement, a third reader was invited to resolve the issue and reach a consensus.

\section{The Definition of ICASB}

The degree of intracranial stenosis on MRA was calculated based on the method from the WASID study [7]. The degree of ICAS was measured in each patient. The atherosclerotic lesions of intracranial vessels on magnetic resonance angiography (MRA) were visually graded: 0 , no stenosis; 1 , stenosis $<50 \%$; 2 , stenosis $50-$ $99 \%$; and 3, occlusion. The assessment of the ICAS location included MCA, anterior and posterior cerebral arteries, basal artery (BA), and intracranial portions of the internal carotid and vertebral arteries. The sum of the involved intracranial vessels was defined as the ICASB according to prior study in the subgroups [8]. The patients were categorized into three groups according to ICASB: (1) <4 scores; (2) 4-5 scores; (3) >5scores.

We estimated the risk of stroke in subgroups of patients categorized according to the time from diffusionweighted imaging to evaluation by a stroke specialist and the number of acute (new) infarction-related lesions (single infarction vs. > multiple infarctions). 


\section{Outcome Measures}

The primary outcome was progressive deterioration or recurrence of IS in 1 year. The definition of progressive deterioration of IS had worsened by $\geq 4$ points of the initial NIHSS score from the index stroke, a new focal neurological deficit of vascular origin lasting for $>24 \mathrm{~h}$ was defined as stroke recurrence. Patients or their authorized proxies were contacted over the telephone at 3,6 , and 12 months after discharge, in order to monitor whether patients experienced new symptoms or were hospitalized again because of another stroke; these data were collected by trained research personnel at Beijing Tian Tan hospital. In addition, the recurrence or progressive deterioration of IS were verified based on the NIHSS score and the presence of new neurological deficits documented in the medical records. The patients' medical documents were reviewed by an experienced stroke neurologist to ensure a reliable diagnosis of recurrence or progressive deterioration of IS. In case of missing any personal information or dead without hospitalization, a stroke neurologist or investigators made the decision based on the medical record. Any death was verified by examining the hospital medical records or local citizen registry. At discharge and 1 year after stroke onset, the daily activities were assessed by mRS [9]. The functional dependence was defined as $m R S>2$. The stroke severity at admission and discharge was measured by the NIHSS [10] based on the stroke onset.

Table 1. Baseline Characteristics of the Participants.

\begin{tabular}{|c|c|c|c|c|c|}
\hline Characteristics & $\begin{array}{c}\text { Total, N }(\%) \\
2864\end{array}$ & $\begin{array}{c}\text { ICASB }(<4) \\
(n=2401)\end{array}$ & $\begin{array}{c}\text { ICASB (4-5) } \\
(n=214)\end{array}$ & $\begin{array}{c}\text { ICASB }(>5) \\
(n=150)\end{array}$ & $p$-value \\
\hline \multicolumn{6}{|l|}{ Demographic } \\
\hline Male, n (\%) & $1944(67.9)$ & $1706(68.5)$ & $138(64.4)$ & $100(62.8)$ & 0.19 \\
\hline Age (mean \pm SD), year & $61.91 \pm 11.2$ & $61.71 \pm 11.3$ & $63.24 \pm 10.4$ & $63.2 \pm 11.0$ & 0.06 \\
\hline \multicolumn{6}{|l|}{ Vascular risk factors } \\
\hline Diabetes mellitus, n (\%) & $699(24.7)$ & $569(23.0)$ & $77(36.7)$ & $53(34.6)$ & $<0.0001$ \\
\hline Hypertension, n (\%) & $1898(66.8)$ & $1612(65.2)$ & $150(75.0)$ & $127(81.4)$ & $<0.0001$ \\
\hline Hyperlipidemia, n (\%) & $450(18.2)$ & $378(17.5)$ & $37(21.4)$ & $35(25.7)$ & 0.0295 \\
\hline Family history of stroke, n (\%) & $296(10.5)$ & $236(9.6)$ & $34(15.1)$ & $26(16.3)$ & 0.0006 \\
\hline previous and current smoker, n (\%) & $1296(45.8)$ & $1138(46.2)$ & $91(43.3)$ & $67(42.9)$ & 0.55 \\
\hline History of IS, n (\%) & $710(24.8)$ & $575(23.1)$ & $62(29.0)$ & $73(45.9)$ & $<0.0001$ \\
\hline History of hemorrhage stroke, $\mathrm{n}(\%)$ & $54(1.1)$ & $46(1.9)$ & $3(1.4)$ & $25(3.1)$ & 0.44 \\
\hline Heart disease, n (\%) & $228(7.9)$ & $186(7.7)$ & $26(12.2)$ & $16(10.1)$ & 0.03 \\
\hline Peripheral vascular disease, $\mathrm{n}(\%)$ & $21(0.7)$ & $16(0.6)$ & $4(1.9)$ & $1(0.6)$ & 0.13 \\
\hline Heavy drinker, n (\%) & $407(14.2)$ & $355(14.3)$ & $32(14.9)$ & $20(12.6)$ & 0.79 \\
\hline Severity of ICAS & & & & & $<0.001$ \\
\hline None & $1551(54.2)$ & $1551(62.3)$ & 0 & 0 & \\
\hline$<50 \%$ & $393(13.7)$ & $390(15.7)$ & $3(1.4)$ & 0 & \\
\hline $50-99 \%$ & $505(17.6)$ & $338(13.6)$ & $123(57.5)$ & $44(27.7)$ & \\
\hline $100 \%$ & $415(14.5)$ & $212(8.5)$ & $88(41.1)$ & $115(72.3)$ & \\
\hline \multicolumn{6}{|l|}{ Performance measures } \\
\hline Antihypertension, n (\%) & $1392(48.7)$ & $1191(47.9)$ & $109(51.6)$ & $92(57.9)$ & \multirow{2}{*}{$\begin{array}{l}0.04 \\
0.0065\end{array}$} \\
\hline Early antithrombotic, n (\%) & $2494(87.0)$ & $2186(87.7)$ & $177(82.7)$ & $32(20.1)$ & \\
\hline Statins, n (\%) & $2170(75.8)$ & $1860(74.7)$ & $180(84.1)$ & $130(81.8)$ & 0.0016 \\
\hline NIHSS score at admission(IQR) & $4(1-7)$ & $3(1-6)$ & $4(2-8)$ & $5(2-10)$ & 0.0001 \\
\hline Pre-admission $\mathrm{MRS}<2$ scores, $\mathrm{n}(\%)$ & $2857(100)$ & $2484(87.0)$ & $213(7.5)$ & $159(5.6)$ & 0.02 \\
\hline
\end{tabular}


Table 2. Univariate analysis of outcome with recurrent stroke at 12 months.

\begin{tabular}{|c|c|c|c|c|}
\hline Characteristics & $\begin{array}{c}\text { Total } \\
(\mathrm{n}=2864)\end{array}$ & $\begin{array}{c}\text { Recurrent stroke } \\
(\mathbf{n}=\mathbf{1 0 0})\end{array}$ & $\begin{array}{c}\text { No-recurrent stroke } \\
(n=2764)\end{array}$ & p-value \\
\hline Male sex & $1944(67.9)$ & $84(64.6)$ & $1860(68.0)$ & 0.41 \\
\hline Age (mean $\pm S D)$, year & $61.91 \pm 11.2$ & $65.47 \pm 10.8$ & $61.74 \pm 11.2$ & 0.0002 \\
\hline Diabetes mellitus, n (\%) & $699(24.7)$ & $42(32.8)$ & $657(24.3)$ & 0.03 \\
\hline Hypertension, n (\%) & $1898(66.8)$ & $97(74.6)$ & $1801(66.4)$ & 0.05 \\
\hline Hyperlipidemia, n (\%) & $450(18.2)$ & $17(15.6)$ & $433(18.4)$ & 0.46 \\
\hline Familyhistory of stroke, n (\%) & $296(10.5)$ & $21(16.8)$ & $275(10.2)$ & 0.02 \\
\hline Previousandcurrent smoking, n (\%) & $1296(45.8)$ & $45(34.9)$ & $1251(46.3)$ & 0.01 \\
\hline Heavy drinker, $\mathrm{n}(\%)$ & $407(14.2)$ & $13(10.0)$ & $394(14.4)$ & 0.16 \\
\hline History of IS, n (\%) & $710(24.8)$ & $50(38.5)$ & $660(24.1)$ & 0.0001 \\
\hline History of hemorrhage stroke, n (\%) & $54(1.9)$ & $1(0.8)$ & $53(1.9)$ & 0.34 \\
\hline History of heart disease, $\mathrm{n}(\%)$ & $228(8.0)$ & $19(14.6)$ & $209(7.6)$ & 0.004 \\
\hline Peripheral vascular disease, $\mathrm{n}(\%)$ & $21(0.7)$ & $1(0.8)$ & $20(0.7)$ & 0.96 \\
\hline Body mass index & $24.58 \pm 3.2$ & $24.62 \pm 2.4$ & $24.57 \pm 3.2$ & 0.87 \\
\hline $\mathrm{SBP}$ at admission, $\mathrm{mmHg}$ & $150.7 \pm 23.4$ & $155.26 \pm 22.9$ & $150.49 \pm 23.4$ & 0.03 \\
\hline DBP at admission, $\mathrm{mmHg}$ & $87.73 \pm 13.4$ & $89.72 \pm 12.6$ & $87.62 \pm 13.4$ & 0.09 \\
\hline NIHSS score at admission & $4(1-7)$ & $5(2-10)$ & $3(1-7)$ & $<0.0001$ \\
\hline Pre-admission $\mathrm{mRS}<2$ scores, $\mathrm{n}(\%)$ & $2857(100)$ & $128(4.5)$ & $2729(95.5)$ & 0.03 \\
\hline \multicolumn{5}{|l|}{ Performance Measures } \\
\hline Early antithrombotic therapy, n (\%) & $367(12.8)$ & $25(19.4)$ & $3425(12.5)$ & 0.022 \\
\hline Statins, $\mathrm{n}(\%)$ & $2170(75.8)$ & $93(71.5)$ & $2077(76.0)$ & 0.25 \\
\hline Antihypertension, n (\%) & $1392(48.7)$ & $67(51.9)$ & $1341(48.6))$ & 0.45 \\
\hline Severity of ICAS & & & & 0.0006 \\
\hline None & $1551(54.2)$ & $51(39.2)$ & $1500(54.9)$ & \\
\hline$<50 \%$ & $393(13.7)$ & $20(15.4)$ & $373(13.6)$ & \\
\hline $50-99 \%$ & $505(17.6)$ & $26(20.0)$ & $479(17.5)$ & \\
\hline $100 \%$ & $415(14.5)$ & $33(25.4)$ & $382(14.0)$ & \\
\hline ICASB & & & & $<0.0001$ \\
\hline ICASB $(<4)$ & $2491(87.0)$ & $97(74.6)$ & $2394(87.6)$ & \\
\hline ICASB (4-5) & $214(7.5)$ & $22(16.9)$ & $192(7.0)$ & \\
\hline ICASB $(>5)$ & 159(1.9) & $11(8.5)$ & $148(5.4)$ & \\
\hline Category of infarction & & & & 0.038 \\
\hline Single infarction & $1864(86.5)$ & $87(79.8)$ & $1777(86.8)$ & \\
\hline Multiple infarctions & $292(13.5)$ & $22(20.2)$ & $270(13.2)$ & \\
\hline mRS at 12 months (3-6) & $507(19.0)$ & $70(56.0)$ & $437(17.1)$ & $<0.0001$ \\
\hline
\end{tabular}

DBP, diastolic BP; SBP, systolic BP; ICASB, intracranial atherosclerosis burden; NIHSS, National Institutes of Health Stroke Scale.

* Continuous variables are expressed as mean $\pm \mathrm{SD}$; other values are expressed as $\mathrm{n}(\%)$.

\section{Statistical Methods}

Continuous variables with non-normal distribution were summarized as median (interquartile range). The categorical variables such as male sex and vascular risk factors were presented as absolute numbers and percentages. The comparison of continuous variables utilized independent samples t-test or Wilcoxon test, whereas the $\chi^{2}$ test or Fisher's exact test was used for the comparison of categorical variables. The adjusted differences in NIHSS score at admission and hospital stay for patients' single and multiple infarctions were analyzed by the general linear model. The univariate and multivariate Cox proportional hazards regression models 
were used to estimate the impact of the hazard ratios (HR) of the putative determinants of recurrent stroke. All probability values were 2 -tailed; $P<0.05$ was considered statistically significant. All analyses were performed using SAS version 9.1 (SAS Institute, Cary, NC, USA).

\section{RESULTS}

The cohort consisted of 2864 consecutive patients from October 2007 to June 2009. The follow-up was continued for 12 months; $176(6.2 \%)$ patients were lost to followup.

Baseline of the clinical features of the participants were summarized in Table 1. The mean age at admission for the index stroke was $61.9 \pm 11.2$ years, and $67.9 \%$ of the patients were male. Hypertension (66.8\%), hyperlipidemia (18.2\%), diabetes mellitus (24.7\%), and history of cerebral ischemia $(24.8 \%)$ were the most common vascular risk factors. During the hospital stay, the common treatments included early antithrombotic therapy $(87.0 \%)$, statins $(75.8 \%)$, and antihypertensive drugs (48.7\%). According to the three groups of ICAS burden, patients with high ICAS burden were likely to be older and had risk factors such as diabetes mellitus, hypertension, family history of stroke, and history of cerebral ischemia. The admission NIHSS score was high in the groups of high ICASB $(P<0.0001)$. The severity of intracranial artery stenosis differs associating with burden degrees, indicating in those with $>4$ scores obtained more proportion over 50\% ICAS $(P<0.0001)$. Moreover, $\mathrm{mRS}$ score at before admission was low in the groups of higher ICASB $(P=0.02)$.

Table 3. Multivariate analysis for predictors of recurrent stroke at 12 months.

\begin{tabular}{llc}
\hline Predictors & HR $(\mathbf{9 5 \%} \mathbf{C l})$ & $\boldsymbol{p}$-value \\
\hline ICASB (4-5) & $1.96(1.08-3.56)$ & 0.027 \\
Family history of stroke & $2.03(1.21-3.41)$ & 0.0072 \\
History of ((IS) & $1.76(1.14-2.73)$ & 0.0096 \\
History of heart history & $1.40(0.74-2.67)$ & 0.298 \\
NIHSS score at admission & $1.05(1.00-1.09)$ & 0.028 \\
\hline CI indicates confidence interval; HR, hazard ratio; ICASB, intracranial \\
atherosclerosis burden; NIHSS, National Institutes of Health Stroke \\
Scale; IS, ischemic stroke.
\end{tabular}

Univariate analysis showed that several factors were associated with recurrent stroke (Table 2). The family history of stroke, history of cerebral ischemia, heart disease, previous or current smoking and high NIHSS at admission, the moderate or severe artery stenosis $(>50 \%)$ were correlated to recurrent stroke $(P<0.05)$. Moreover, patients with ICASB $>4$ scores showed a significantly higher rate of recurrent stroke $(P<0.0001)$. For the lesion category, group with multiple infarctions were more likely associated with the risk of recurrence than the single infarction group $(P=0.038)$. In multivariate analysis, ICASB (4-5) (HR: 1.96; 95\% CI: 1.08-3.56; $P=0.027$ ), family history of stroke (HR: 2.03 ; $95 \% \mathrm{CI}$ : $1.21-3.41 ; P=0.0072$ ), history of cerebral ischemia (HR: 1.76 ; $95 \%$ CI: $1.14-2.73 ; P=0.0096)$ were correlated to the recurrence of stroke. Besides, NIHSS score at admission, family history of stroke and history of ischemic stroke were independent predictors for recurrent stroke as well (Table 3).

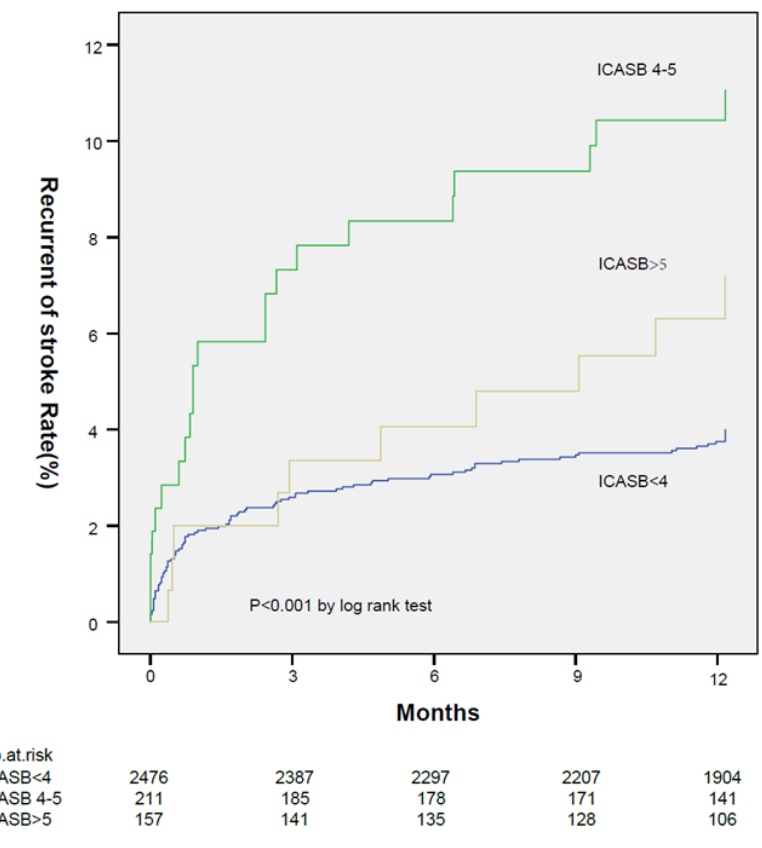

Figure 1. Kaplan-Meier curves for stroke recurrence from the time of the qualifying event up to 1 year in the three groups. ICASB, intracranial atherosclerosis burden.

Kaplan-Meier analysis showed that the ICASB (45) groups was associated with recurrent stroke at12 months as compared to the ICASB $<4$ scores group and $>5$ scores (Fig. 1). The proportion of single and multiple infarction lesions differs with different ICASB, higher percentage of multiple lesions in the groups of higher burden $>4$ scores $(P<0.001)$ (Fig. 2$)$.

\section{DISCUSSION}

We evaluated outcomes of the patients with the different ICASB scores. ICASB can be assessed by different methods. One of prior study [3] calculated ICASB by evaluating intracranial only, concurrent extra-intracranial lesions, extracranial only, recent study [11] calculated 
ICASB according to the numbers of artery stenosis which relative to clinical outcomes. In our study, most of traditional vascular risk factors such as diabetes mellitus, hypertension, family history of stroke, and history of cerebral ischemia were associated with higher ICASB ( $\mathrm{P}<0.0001)$, and NIHSS score at admission was higher in the groups of higher ICASB $(\mathrm{P}<0.0001)$, and the ratio of $\mathrm{mRS}$ score $<2$ at pre-admission was lower in the groups of higher ICASB $(\mathrm{P}=0.02)$. In the WarfarinAspirin Systematic Intracrinal Disease (WASID) study [12], this variety of symptomatic intracranial stenosis was independently related to a higher risk of subsequent stroke in the territory of the stenotic artery. The risk of recurrent stroke in the territory of the symptomatic stenotic artery was as high as $23 \%$ during the first year in patients with stenosis $\geq 70 \%$. The current study showed that patients with ICASB ( $>4$ scores) had a significantly high risk of recurrent stroke, and ICASB (>4 scores), family history of stroke, history of cerebral ischemia and NIHSS score at admission were independent predictors of recurrent stroke. Moreover, the proportion of single and multiple infarction lesions differs with different ICASB, the higher of burden, more of multiple lesions.

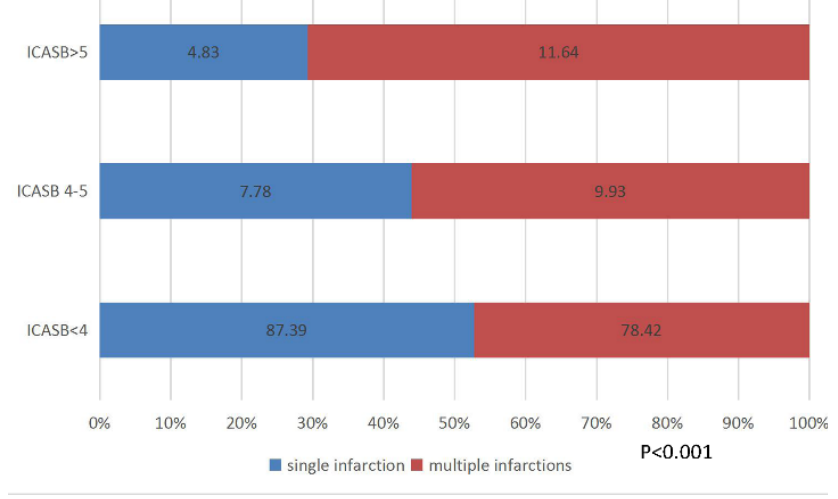

Figure 2. Proportion of infarction lesions in different ICSB group. Multiple infarctions were seen more than single infarction in group ICASB $>4$ scores $(\mathrm{P}<0.001)$. $\mathrm{P}$-value was obtained by Chi-square test to assess the association of intracranial atherosclerosis burden (ICASB) in single infarction and multiple infarctions.

The current study showed that the ICASB (45 scores) group were more associated with recurrent stroke in 12 months compared to the ICASB <4 scores and $>5$ group showing to a large number of $50-99 \%$ stenosis arteries in the group, and also result from little samples in $>5$ group. Thus, single infarction and multiple infarctions might have different ICASB, the higher portion of multiple infarction lesions were found in group with higher ICASB scores, this more relative to most of clinic interpretations of multiple lesion, showing high risk of embolism and recurrence, and this observation was in agreement with the previous study [6].

Moreover, our study show high stroke recurrence at 12 months among patients with severe stenosis, which were indicated the same results in the most recently RCTs of WASID [12] and SAMPRIS [13]. ICAS of $<50 \%$ and $50-69 \%$ luminal stenosis is usually regarded as nonsignificant or defined mild or moderate lesions respectively [12, 14], however, ICASB (4-5) group was highly related to recurrent stroke in our study, and there were more patients with mild stenosis in this groups. Therefore, recurrent risks in patients with mild stenosis need to be fully appreciated in future studies, so that highrisk patients would not be missed because of artificially graded severity of ICAS by the degree of luminal stenosis [13]. And then, different stroke mechanisms accompany with ICAS, and different aetiological mechanisms has significant clinical implications [15]. Recently study show Interleukin-33 (IL-33) and levels of fibrinogen after anticoagulation were related to ischemic stroke $[16,17]$. so the evaluation of intracranial atherosclerotic disease should be reconsidered from grading of stenosis to hemodynamic and emboligenic lesion characterization because of a large mountain of evidence about collaterals, hemodynamic impact, and other factors in determining subsequent stroke risk in patients with symptomatic ICAS [18-20], so we should optimally identify those who are truly at high risk.

The study had several limitations. First, the intracranial large artery was evaluated by MRA. Although it was flow-sensitive and not as accurate as DSA, it is difficult to differ vascular stenosis from 50$70 \%$ to $70-99 \%$. However, MRA was noninvasive and easily accessible as compared to DSA [21]. Second, we could not accurately exclude a reanalyzing embolus from an in situ stenosis although the patients with presumed cardio-embolism were excluded. Third, intracranial vasculitis or primary agilities of the central nervous system (PACNS) were not excluded specifically. Fourth, we did not record the changes in medications during the 12 months follow-up period.

In conclusion, we found that ICASB was associated with recurrent stroke. Multiple infarctions had higher ICASB scores for symptomatic intracranial artery stenosis patients. Except severity of stenosis, this might be providing an additional assessment method for mechanism and prognosis of sICAS patients.

\section{Acknowledgments}

The authors thank all participating hospitals, colleagues, nurses, and imaging and laboratory technicians. The study is supported by grants from the Ministry of Science 
and Technology of the People's Republic of China (2006BAI01A11, 2011BAI08B01, 2011BAI08B02, 2012ZX09303-005-001, and 2013BAI09B03), a grant from the Beijing Biobank of Cerebral Vascular Disease (D131100005313003), a grant from Beijing Institute for Brain Disorders (BIBD-PXM2013_014226_07_000084) and grants from the National Natural Science Foundation of China (No. 81322019 and No.81601015).

\section{References}

[1] Gorelick PB, Wong KS, Bae HJ, Pandey DK (2008). Large artery intracranial occlusive disease: a large worldwide burden but a relatively neglected frontier. Stroke, 39:2396-2399.

[2] Sacco RL, Kargman DE, Gu Q, Zamanillo MC (1995). Race-ethnicity and determinants of intracranial atherosclerotic cerebral infarction. The Northern Manhattan Stroke Study. Stroke, 26:14-20.

[3] Lau AY, Wong KS, Lev M, Furie K, Smith W, Kim AS (2013). Burden of intracranial steno-occlusive lesions on initial computed tomography angiography predicts poor outcome in patients with acute stroke. Stroke, 44:1310-1316.

[4] Kasner SE, Chimowitz MI, Lynn MJ, Howlett-Smith H, Stern BJ, Hertzberg VS, et al. (2006). Predictors of ischemic stroke in the territory of a symptomatic intracranial arterial stenosis. Circulation, 113:555-563.

[5] Wang Y, Zhao X, Liu L, Soo YO, Pu Y, Pan Y, et al. (2014). Prevalence and outcomes of symptomatic intracranial large artery stenoses and occlusions in China: the Chinese Intracranial Atherosclerosis (CICAS) Study. Stroke, 45:663-669.

[6] Amarenco P, Lavallee PC, Labreuche J, Albers GW, Bornstein NM, Canhao P, et al. (2016). One-Year Risk of Stroke after Transient Ischemic Attack or Minor Stroke. N Engl J Med, 374:1533-1542.

[7] Samuels OB, Joseph GJ, Lynn MJ, Smith HA, Chimowitz MI (2000). A standardized method for measuring intracranial arterial stenosis. AJNR Am J Neuroradiol, 21:643-646.

[8] Jeon SB, Chun S, Choi-Kwon S, Chi HS, Nah HW, Kwon SU, et al. (2012). Biomarkers and location of atherosclerosis: matrix metalloproteinase- 2 may be related to intracranial atherosclerosis. Atherosclerosis, 223:442-447.

[9] Sulter G, Steen C, De Keyser J (1999). Use of the Barthel index and modified Rankin scale in acute stroke trials. Stroke, 30:1538-1541.
[10] DeGraba TJ, Hallenbeck JM, Pettigrew KD, Dutka AJ, Kelly BJ (1999). Progression in acute stroke: value of the initial NIH stroke scale score on patient stratification in future trials. Stroke, 30:1208-1212.

[11] Kim YD, Choi HY, Cho HJ, Cha MJ, Nam CM, Han SW, et al. (2010). Increasing frequency and burden of cerebral artery atherosclerosis in Korean stroke patients. Yonsei Med J, 51:318-325.

[12] Chimowitz MI, Lynn MJ, Howlett-Smith H, Stern BJ, Hertzberg VS, Frankel MR, et al. (2005). Comparison of warfarin and aspirin for symptomatic intracranial arterial stenosis. N Engl J Med, 352:1305-1316.

[13] Chimowitz MI, Lynn MJ, Derdeyn CP, Turan TN, Fiorella D, Lane BF, et al. (2011). Stenting versus aggressive medical therapy for intracranial arterial stenosis. N Engl J Med, 365:993-1003.

[14] Zhang C, Wang Y, Zhao X, Wang D, Liu L, Wang C, et al. (2014). Distal single subcortical infarction had a better clinical outcome compared with proximal single subcortical infarction. Stroke, 45:2613-2619.

[15] Tian C, Cao X, Wang J (2017). Recanalisation therapy in patients with acute ischaemic stroke caused by large artery occlusion: choice of therapeutic strategy according to underlying aetiological mechanism? Stroke and Vascular Neurology, 2:244-250.

[16] Qian L, Yuanshao L, Wensi H, Yulei Z, Xiaoli C, Brian W, et al. (2016). Serum IL-33 Is a Novel Diagnostic and Prognostic Biomarker in Acute Ischemic Stroke. Aging Dis, 7:614-622.

[17] Liu J, Wang D, Xiong Y, et al. (2017) Association between Coagulation Function and Cerebral Microbleeds in Ischemic Stroke Patients with Atrial Fibrillation and/or Rheumatic Heart Disease. Aging Dis, 8;131-135.

[18] Leng X, Wong KS, Liebeskind DS (2014). Evaluating intracranial atherosclerosis rather than intracranial stenosis. Stroke, 45:645-651.

[19] Liebeskind DS, Cotsonis GA, Saver JL, Lynn MJ, Turan TN, Cloft HJ, et al. (2011). Collaterals dramatically alter stroke risk in intracranial atherosclerosis. Ann Neurol, 69:963-974.

[20] Leng XY, Chen XY, Chook P, Xiong L, Lin WH, Liu JY, et al. (2013). Correlation of large artery intracranial occlusive disease with carotid intima-media thickness and presence of carotid plaque. Stroke, 44:68-72.

[21] Man BL, Fu YP, Chan YY, Lam W, Hui CF, Leung WH, et al. (2009). Use of magnetic resonance angiography to predict long-term outcomes of ischemic stroke patients with concurrent stenoses in Hong Kong. Cerebrovasc Dis, 28:112-118. 